\title{
Caracterización sociodemográfica y familiar de obesos intervenidos a través de cirugía bariátrica en el Hospital Universitario de Neiva
}

\author{
Socio-demographic and family characteristics of obese people \\ who underwent bariatric surgery at University Hospital in Neiva
}

\author{
Gloria E. Mejía Rubiano' , Fabio A.Salazar-Piñeros², Luis Eduardo Sanabria R33, \\ Héctor Adolfo Polania Lizcano ${ }^{4}$, Rolando Medina Rojas ${ }^{5}$
}

\section{Resumen}

Objetivo: Caracterizar desde el punto de vista clínico y social a las personas obesas sometidas a cirugía bariátrica en el Hospital Universitario de Neiva en el periodo entre septiembre de 2011 y el mismo mes de 2012. Material y métodos: Se realizó un estudio descriptivo de corte trasversal en el Hospital Universitario Hernando Moncaleano Perdomo de Neiva, aplicando una encuesta telefónica y revisión de historias clínicas a 25 pacientes que fueron intervenidos a través de cirugía bariátrica en la Institución. Se estudiaron las variables: sexo, estrato socioeconómico, ocupación, hábitos alimenticios, índice de masa corporal, características de satisfacción con la condición física y vida antes y después de la cirugía, para determinar características a nivel familiar se aplicó el APGAR familiar. Resultados: En la muestra estudiada existió un predominio del sexo femenino, con edades entre 20 y 53 años, con pareja estable de estrato socioeconómico bajo, con un nivel de escolaridad hasta secundaria y viven en Neiva. El peso de las personas antes de la cirugía estaba entre $93 \mathrm{Kg}$ y $155 \mathrm{Kg}$. con un índice de masa corporal entre 36 y 59 . Antes de la cirugía no se sentían para nada satisfechos con su condición física y con su vida, actualmente se sienten moderadamente satisfechos. Además son familias nucleares y existe un elevado número de familias con disfuncionalidad leve. Conclusión: Los pacientes presentan malos hábitos alimenticios antes de la cirugía y se evidencio disfuncionalidad familiar.

Palabras claves: Obesidad, hábitos alimenticios, función familiar.

\begin{abstract}
Objective: To make a clinical and social characterization of obese people who underwent bariatric surgery at University Hospital in Neiva, between September 2011 and September 2012. Materials and Methods: A crosssectional, descriptive study was conducted at Hernando Moncaleano Perdomo University Hospital in Neiva. It was made a telephone survey and medical histories were checked for 25 patients who underwent bariatric surgery at this health center.

It was considered the following variables: sex, socioeconomic status, occupation, eating habits, body mass index, characteristics of satisfaction with fitness and lifestyle before and after surgery. In order to determine family characteristics it was carried out APGAR family. Results: Most members of the sample were women whose ages were between 20 and 53. They had a stable partner, belonged to low socioeconomic stratum, had finished high school and lived in Neiva. Weight before surgery was between $93 \mathrm{Kg}$ and $155 \mathrm{Kg}$, with body mass index between 36 and 59. Before surgery they didn't feel at all satisfied with their fitness and their life. At present they feel moderately satisfied. In addition, their families are nuclear and a high percentage of them are mild dysfunctional. Conclusion: Patients have bad eating habits before surgery and it was shown family dysfunction.
\end{abstract}

Keywords: Obesity, eating habits, family function.

1. Psicóloga Especialista en epidemiología, Universidad Surcolombiana.

2. Psicólogo clínico, profesor universidad Surcolombiana, Neiva.

3. MD. Cirujano General Fellow UCI-FSFB. Coordinador Departamento de Cirugía USCO-HUN. Profesor Asistente, Departamento de Ciencias Clínicas, Facultad de Salud, Universidad Surcolombiana, Neiva, Colombia.

4. MD. Gastroenterólogo Clínico Quirúrgico. Departamento de Cirugía, Hospital Universitario de Neiva, Profesor Asistente. Jefe Departamento de Ciencias Clínicas. Universidad Surcolombiana, Neiva, Colombia.

5. MD. Cirujano General, Hospital Universitario de Neiva, Colombia.

Datos de contacto: Gloria Esperanza Mejía Rubiano. Correo electrónico: gloria.e.mejia@hotmail.com.

Recibido: 21/05/2013 - Revisado: 09/09/2013 - Aceptado: 24/06/2014 


\section{Introduccion}

Según la OMS el sobrepeso y la obesidad se definen como una acumulación anormal o excesiva de grasa que puede ser perjudicial para la salud ${ }^{[1]}$. La obesidad es un factor de riesgo para enfermedades crónicas como: enfermedades cardiacas, diabetes mellitus tipo 2, hipertensión arterial, ictus y algunos tipos de cáncer, entre otras. La evidencia sugiere que se trata de una enfermedad con origen multifactorial: genético, ambiental, psicológico entre otros, que genera una acumulación excesiva de grasa con hipertrofia general del tejido adiposo. La obesidad es indiferente de raza, edad, nivel socio económico, sexo o ubicación geográfica.

El sobrepeso y la obesidad son el quinto factor principal de riesgo de defunción en el mundo. Anualmente se presentan 2,8 millones de decesos en personas con sobrepeso u obesidad. Además, el $44 \%$ de la carga de diabetes mellitus tipo II, el $23 \%$ de la carga de cardiopatías isquémicas y entre el $7 \%$ y el $41 \%$ de la carga de algunos cánceres son atribuibles al sobrepeso y la obesidad [1].

En Colombia se calcula que existen 12 millones de personas con sobrepeso u obesidad, aproximadamente un millón de personas con diabetes y dos millones de individuos con hiperglicemia de ayuno o intolerancia a los carbohidratos, entidades que preceden a la diabetes tipo 2. La magnitud de problemas es incalculable ${ }^{[2]}$.

La prevalencia de la obesidad está aumentando rápidamente y se asocia con comorbilidades importantes. En la actualidad, la evidencia apoyo el uso de la pérdida de peso con procedimientos quirúrgicos modernos cuidadosamente seleccionados en los pacientes con obesidad extrema. Y esto es logrado por la cirugía bariátrica $(\mathrm{CB})$.

Entre otras aplicaciones, actualmente la $\mathrm{CB}$ se recomienda a pacientes con obesidad extrema. La familia juega un rol muy importante en cuanto al aumento en la obesidad o al descenso de la misma.

$\mathrm{La} \mathrm{CB}$ es una rama de la cirugía destinada a realizar, mediante diversas operaciones, modificaciones en el aparato digestivo a fin de reducir la capacidad gástrica, asociada o no a la disminución de la absorción de los nutrientes ingeridos. Bariátrica, deriva de vocablos griegos que significan Medicina o Cirugía del Peso. Esta especialización data de mediados de los años 1970 y en los Estados Unidos existe una Sociedad Científica desde $1983^{[3]}$. El Hospital Universitario de Neiva Hernando Moncaleano Perdomo implemento la CB desde el año 2011.

Por todo lo anterior es importante saber cuáles son las condiciones sociodemográficas y familiares del obesos que se realiza la $\mathrm{CB}$ en el Hospital Universitario Neiva y de esta manera identificar cuáles son las mayores falencias y necesidades que tiene esta población, de esta forma elaborar estrategias para que el grupo multidisciplinario de CB de la Institución, tengan más herramientas para brindar mayor bienestar y seguridad al paciente y su familia.

Teniendo como objetivo establecer las características sociodemográficas y familiares de los Obesos Intervenidos con CB, en el Hospital Universitario de Neiva. Con el fin de enriquecer el conocimiento sociodemográfico y familiar en esta población.

\section{Materiales y métodos}

Se realizó un estudio cuantitativo descriptivo observacional de corte transversal se aplicaron dos encuestas a los pacientes que participaron en el estudio; la primera el APGAR (Appearance; Pulse; Grimace; Activity; Respiration) familiar, para evaluar posible difusión familiar; y la segunda para valorar los hábitos alimenticios antes y después de la cirugía, la condición física y de vida y características familiares, acompañado de un formato de datos básicos que fueron extraídos de la historia clínica de los pacientes que se intervinieron con la cirugía bariátrica en el Hospital Universitario de Neiva de septiembre de 2011 a septiembre de 2012.

El investigador fue la persona quien aplico el instrumento a todos los pacientes, la forma en que fueron abordados para responder la encuesta fue vía telefónica, los 25 pacientes que participaron fueron orientados sobre las implicaciones del estudio, la confidencialidad de la información y su participación fue voluntaria sin ninguna clase de presión.

Los pacientes encuestados fueron elegidos de forma no probabilística, no representativa por conveniencia esto hace referencia a que no hay tamaño de muestra por que no se va a comparar o a inferir sobre el universo, se tomó a toda la población que se intervino con la cirugía bariátrica de septiembre de 2011 a septiembre de 2012 .

Los criterios de inclusión fueron: pacientes sometidos a $\mathrm{CB}$ en el Hospital Universitario de Neiva entre septiembre de 2011 y septiembre de 2012, mayor de edad y voluntad para participar en el estudio, como criterios de exclusión se tuvieron: La no participación voluntaria, pacientes intervenidos en otro tiempo fuera del establecido.

Los instrumentos elaborados se sometieron a un pilotaje esto se realizó con el fin de evaluar si la encuesta estaba diseñada a cumplir con el objetivo propuesto para la investigación, el instrumento fue revisado por un comité de expertos esto se efectuó para que el instrumento tuviera mayor validez.

Es de aclarar que el estudio presenta sesgos de medición al desarrollar la encuesta por teléfono y sesgos de memoria al realizar preguntas de actividades que realizaban las personas un año antes de la cirugía ese fue un limitante en el estudio.

Las variables estudiadas en cada paciente se encuentran: edad, sexo, estado civil, nivel educativo, ocupación, procedencia, estrato socioeconómico, seguridad social, peso, talla, índice de masa corporal (IMC), comorbilidades, hábitos alimenticios, condición física, condición de vida, función familiar.

La información fue organizada en el programa de EXCEL versión 2010 e importada al programa SPSS 15 para el respectivo análisis; para cada variable se hizo un análisis univariado con medidas de frecuencia.

El estudio se realizó previa autorización del comité de ética del Hospital Universitario de Neiva. Según la Resolución número 8430 de 1993, artículo 11, se clasifica como investigación sin riesgo debido a que la técnica y el método de investigación fue documental, considerándose la revisión de historias clínicas y aplicación de entrevistas sin que se identifiquen ni se traten aspectos sensitivos de la conducta.

\section{Resultados}

Las características sociodemográficas de la muestra son presentadas en la Tabla 1. Se encontró que por cada 7 mujeres intervenidas hay un hombre. La edad de los pacientes se encuentra entre 20 y 53 años (Rango=33 Años); la edad promedio de estos pacientes es de 36 años $( \pm 8,8)$

En su mayoría los pacientes poseen pareja estable, principalmente pertenecen al estrato socioeconómico 2 , y provienen del área urbana. Están afiliados principalmente a la EPS Comfamiliar.

El 64\% de las personas intervenidas con la cirugía bariátrica se desempeña en el Hogar son amas de casa. El número de hijos de los 
Tabla 1: Características Sociodemográficas de los pacientes obesos intervenidos con cirugía bariátrica en el Hospital Universitario Hernando Moncaleano Perdomo de la ciudad de Neiva.

\section{Frecuencia Porcentaje}

\begin{tabular}{lcc}
\hline Genero & & \\
0 Mujer & 22 & 88 \\
1 Hombre & 3 & 12 \\
Total & 25 & 100 \\
\hline
\end{tabular}

\begin{tabular}{lcc}
\hline Estado civil & & \\
Soltero & 2 & 8 \\
Casado & 7 & 28 \\
U. Libre & 11 & 44 \\
Separado & 4 & 16 \\
Viudo & 1 & 4 \\
Total & 25 & 100 \\
\hline
\end{tabular}

\begin{tabular}{lcc}
$\begin{array}{l}\text { Procedencia } \\
\text { Urbana }\end{array}$ & & \\
Rural & 19 & 76 \\
Total & 6 & 24 \\
\hline Estrato económico & 25 & 100 \\
1 & & \\
2 & 5 & 20 \\
3 & 18 & 72 \\
4 & 1 & 4 \\
Total & 1 & 4 \\
\hline
\end{tabular}

\section{Sssgs}

Asmet Salud

12

Cafesalud

Caprecom

Confamiliar

Nueva EPS

Total

312

2

8

2

11

8

44

7

28

Escolaridad

B. primaria

Secundaria

Técnico

Profesional

25

100

Total

$\begin{array}{cc}7 & 28 \\ 13 & 52 \\ 1 & 4 \\ 4 & 16 \\ 25 & 100\end{array}$

\section{Ocupación}

Profesional

Empleado

Hogar

Total

$\begin{array}{cc}4 & 16 \\ 5 & 20 \\ 16 & 64 \\ 25 & 100\end{array}$

Número de hijos

$\begin{array}{lcc}0 & 3 & 12 \\ 1 & 4 & 16 \\ 2 & 9 & 36 \\ 3 & 7 & 28 \\ 4 & 1 & 4 \\ 5 & 1 & 4 \\ \text { Total } & 25 & 100\end{array}$

pacientes obesos intervenidos con cirugía bariátrica se encuentra de la siguiente manera el $36 \%$ tiene 2 hijos y el $28 \% 3$ hijos. Por lo tanto el promedio de hijos que tienen las personas que se realizaron la cirugía son dos. Por otro lado la escolaridad de las personas intervenidas en un $52 \%$ son bachilleres el $28 \%$ tienen básica primaria. Por lo tanto su nivel de escolaridad es mínimo. (Tabla 1)

El 48\% de las personas que participaron en el estudio tienen una familia nuclear (papas e hijos) el 36\% viven en familia extensa (papas, hijos, hermanos, cuñadas, sobrinos, abuelos etc.).

Según lo manifestado por los pacientes en su percepción de sobrepeso o gordura de algún familiar es lo siguiente: el $24 \%$ sus padres presentan o presentaron sobrepeso o gordura igual porcentaje sus hermanos $24 \%$ el $16 \%$ son los hijos los que presenta problema de sobrepeso el $20 \%$ es otro familiar como tíos, abuelos, sobrinos.

El peso antes de la cirugía de las personas que participaron en el estudio estuvo entre los $93 \mathrm{~kg}$ y los $155 \mathrm{~kg}$ (Rango $=62 \mathrm{~kg}$ ) el promedio del peso antes de la cirugía estuvo en $123 \mathrm{~kg}$ con una desviación típica $( \pm 17)$

El Índice de Masa Corporal (IMC) antes de la Cirugía estuvo entre los 36 y 59 IMC $($ Rango= 23$)$ el promedio del IMC de antes de la Cirugía fue de $47( \pm 5,0)$ entonces se puede concluir que el IMC antes de la cirugía de las personas que participaron en este estudio es homogéneo. (Tabla 2)

Las comorbilidades que más se presentaron en la personas que participaron en el estudio fue la gastritis con un $56 \%$ seguido de la diabetes con un $44 \%$ la HTA en un $32 \%$. Los antecedentes familiares de las comorbilidades en los pacientes intervenidos a $\mathrm{CB}$ según lo encontrado en la historia clínica fue lo siguiente: un $48 \%$ preceden de Hipertensión Arterial, el 44\% de obesidad como diabetes, y un $24 \%$ de cáncer.

Según en la revisión de historias clínicas el 32\% de las personas que participaron en el estudio presentan ingesta de gran comilón que está relacionada con la ingesta de atracones de comida, un $24 \%$ presentan múltiple ingesta de alimentos hipercalórico y un $12 \%$ ingesta de alimentos grasos el $28 \%$ presentan todo lo anterior. (Tabla 3 )

Se halló los hábitos de riesgo (fumar, beber licor malos hábitos alimenticios) según lo manifestado por los pacientes el $92 \%$ de las personas que participaron en el estudio un año antes de la cirugía no fumaban. El mismo porcentaje se encontró actualmente, se puede decir que no habido ningún cambio en cuanto al fumar antes de la cirugía y después. (Tabla 4)

En cuanto al consumo de cerveza según lo manifestado el $68 \%$ de los pacientes antes de la CB no consumían, el 20\% se tomaba entre 1 y 10 unidades por semana. En este momento los pacientes manifiestan que el $88 \%$ no consumen, mientras que el $8 \%$ lo hace ocasionalmente, (festividades).

En el consumo de bebidas carbonatadas antes de la $\mathrm{CB}$ el $36 \%$ de las personas consumía una semanalmente de $275 \mathrm{ml}$, el $32 \%$ no consumía. En la actualidad el $92 \%$ de las personas no consumen este tipo de bebidas. Podemos ver el aumento del porcentaje de las personas que no toman gaseosa antes de la cirugía de un $32 \%$ a un $92 \%$.

Además antes de la CB el $84 \%$ de la población no realizaba ejercicio físico. En la actualidad según lo manifestado por los pacientes después de la $\mathrm{CB}$ el $44 \%$ de la población está haciendo ejercicio de 1-3 veces por semana y solo el $24 \%$ no están haciendo ejercicio, podemos ver cómo ha aumentado el ejercicio físico en los pacientes después de la cirugía el $84 \%$ que antes no realiza ejercicio después de la cirugía solo el $24 \%$ no está realizando ejercicio físico. 
Tabla 2 Distribución del IMC Antes Cirugía de los pacientes obesos intervenidos con cirugía bariátrica en el Hospital Universitario Hernando Moncaleano Perdomo de la ciudad de Neiva.

\begin{tabular}{lcccccccc}
\hline N & Media & Mediana & Moda & Desv. Típ & Varianza & Rango & Mínimo & Máximo \\
\hline 25 & 46,76 & 47 & 48 & 5,036 & 5,036 & 23 & 36 & 59 \\
\hline
\end{tabular}

Tabla 3 Distribución Ingesta Alimentaría de los pacientes obesos intervenidos con cirugía bariátrica en el Hospital Universitario Hernando Moncaleano Perdomo de la ciudad de Neiva.

\begin{tabular}{lcc}
\hline & Frecuencia & Porcentaje \\
\hline Ninguno & 1 & 4 \\
Gran Comilón & 8 & 32 \\
Múltiples ingesta hipercalórico & 6 & 24 \\
$\begin{array}{l}\text { Ingesta Habitual } \\
\quad \text { de alimentos grasos }\end{array}$ & 3 & 12 \\
Todos lo anterior & 7 & 28 \\
\hline Total & 25 & 100 \\
\hline
\end{tabular}

E1 92\% de las personas un año antes de la cirugia consumia harinas como pastas, arroz, pan, papa, yuca todos los dias. en la actualidad según lo manifestado por los pacientes después de la cirugía bariátrica el $36 \%$ consume algunas veces harinas el $28 \%$ sigue consmiendo las harinas todos los dias pero en menor proporcion, se evidencia que ha bajado el consumo de harinas en los participantes del estudio.

Un año antes de la cirugía el $40 \%$ consumían comida chatarra (pizza, hamburguesas, perros calientes etc.) algunas veces. El 20\% del consumo era de 3 a 5 veces a la semana o de 1 a 2 veces por semana. Después de la cirugía bariátrica el consumo de comida chatarra ha disminuido notablemente un $80 \%$ rara vez o nunca ingieren estas comidas.

Un año antes de la cirugía el 96\% consumían carne roja, blanca y pescado todos los días, actualmente según lo manifestado por los pacientes el $92 \%$ tienen un consumo de carne y pescado.

Según lo manifestado por los pacientes un año antes de la cirugía el $36 \%$ consumían algunas veces mantequilla y aceites de origen animal un $30 \%$ de 3 a 5 veces a la semana el $20 \%$ todos los días, después de la cirugía según lo manifestado el $88 \%$ de las personas no consumen mantequilla y aceites de origen animal, podemos ver un declinación en el consumo de mantequilla y aceites de origen animal después de la CB.

Un año antes de la cirugía el 44\% consumían dulces. Una vez realizada la $\mathrm{CB}$ según lo manifestado por los pacientes el consumo de dulces, tortas, postres en un $76 \%$ de la población rara vez o nunca consumen.

El consumo de frutas, verduras y legumbres un año antes de la cirugía el $28 \%$ algunas veces consumían el 24\% todos los días, otro $24 \%$ de 3 a 5 veces por semana, el $4 \%$ rara vez nunca. Actualmente podemos ver el aumento del consumo de frutas verduras y legumbres el $44 \%$ consume verduras todos los días.
Se evidencia que según lo manifestado por los pacientes el nivel de satisfacción de condición física un año antes de la cirugía el $80 \%$ no se sentían para nada satisfecho después de la intervención de ese $80 \%$, el $40 \%$ se siente moderadamente satisfechos y el $24 \%$ se sienten muy satisfecho, solo el $4 \%$ manifestó antes de la cirugía sentirse moderadamente satisfecho con su condición física y actualmente se siente muy satisfecho.

Es importante aclarar que después de la intervención los pacientes no señalaron el nada o el ligeramente satisfecho con su condición física esto quiere decir que en las casillas hay cero. Se aplicó la prueba estadística de $\mathrm{Mc} \mathrm{Nemar}{ }^{5}$ buscando diferencia entre el antes y en el después de la intervención de la CB y se pudo evidenciar un cambio en su condición física estadísticamente significativo p $(0,001)$ (Tabla 5).

Según lo manifestado por los pacientes el $68 \%$ antes de la cirugía se sentía para nada satisfechos con su vida, después de la intervención, el 24\% se sienten moderadamente satisfecho, el 20\% se sienten bastante y muy satisfechos con su vida. El $4 \%$ que antes de la cirugía se sentía moderadamente satisfecho actualmente se siente igual, por lo tanto la cirugía no genero ningún cambio en su condición de vida. (Tabla 6)

Es importante aclarar que después de la intervención los pacientes no señalaron el nada satisfecho con su condición física esto quiere decir que en las casillas hay cero. Se aplicó la prueba estadística de Mcnemar 5 buscando diferencia entre el antes y en el después de la intervención de la $\mathrm{CB}$ en la medida de sentirse satisfecho con su condición de vida y se pudo evidenciar un cambio estadísticamente significativo $\mathrm{p}(0,001)$

Según lo expresado por los pacientes que fueron sometidos a la $\mathrm{CB}$ en el Hospital Universitario de Neiva, presenta el 56\% una prevalencia de disfunción familiar leve esto quiere decir que prestan dificultades familiares y el $44 \%$ es normo funcional que tienen una sana función familiar. (Tabla 7)

\section{Discusión}

La mayoría de las personas intervenidas son mujeres en un $88 \%$, las mujeres constituyen un grupo de especial interés en este estudio debido a que representan la gran mayoría de la muestra, nuestros resultados coinciden con los resultados de diferentes estudios epidemiológicos que sitúan la prevalencia de la obesidad en mujeres por encima de la de los hombres, el alto porcentaje de mujeres en estos estudios puede ser que esté ligado a que las mujeres sienten tener más presión por la sociedad y el consumismo con la idealización de belleza que es la mujer delgada según Jessica A. Erickson ${ }^{[4]}$. Por otro lado Julia Navia considera que el ideal de delgadez no constituye la causa principal por la que se accede al tratamiento. Es el ideal de la "normalidad corporal" el que motiva el acceso a la 
Tabla 4 Características de hábitos de riesgo (fumar, beber licor malos hábitos alimenticios) de los pacientes obesos intervenidos con cirugía bariátrica en el Hospital Universitario Hernando Moncaleano Perdomo de la ciudad de Neiva.

\begin{tabular}{|c|c|c|c|c|}
\hline \multirow[t]{2}{*}{ Variables } & \multicolumn{2}{|c|}{ Antes de la $\mathrm{Cx}$ Bariátrica } & \multicolumn{2}{|c|}{ Después de la $C x$ Bariátrica } \\
\hline & Frecuencias & Porcentajes & Frecuencias & Porcentajes \\
\hline
\end{tabular}

\section{Cuantos cigarrillos fumaba}

No fuma

$\begin{array}{cccc}23 & 92 & 23 & 92 \\ 2 & 8 & 2 & 8 \\ 25 & 100 & 25 & 100\end{array}$

Total

25

100

Cuantas cervezas se tomaba a la semana.

No tomaban

1-10 cervezas

17

68

22

88

Ocasional

5

Total

3

20

12

25

100

1

2

4

8

25

100

\section{Consumo de gaseosa a la semana.}

\begin{tabular}{|c|c|c|c|c|}
\hline 0 & 8 & 32 & 23 & 92 \\
\hline 1 & 9 & 36 & 1 & 4 \\
\hline 2 & 4 & 16 & 0 & 0 \\
\hline 3 & 1 & 4 & 0 & 0 \\
\hline 4 & 1 & 4 & 1 & 4 \\
\hline 5 & 1 & 4 & 0 & 0 \\
\hline 12 & 1 & 4 & 0 & 0 \\
\hline Total & 25 & 100 & 25 & 100 \\
\hline \multicolumn{5}{|c|}{ Frecuencia en que realiza ejercicio físico } \\
\hline Nunca & 21 & 84 & 6 & 24 \\
\hline Todos los días & 1 & 4 & 3 & 12 \\
\hline 4 veces por semana & 2 & 8 & 5 & 20 \\
\hline $1-3$ veces por semana & 1 & 4 & 11 & 44 \\
\hline Total & 25 & 100 & 25 & 100 \\
\hline \multicolumn{5}{|l|}{ Consumo de harinas } \\
\hline Todos los días & 23 & 92 & 7 & 28 \\
\hline $3-4$ veces por semana & 2 & 8 & 3 & 12 \\
\hline $1-2$ veces por semana & 0 & 0 & 1 & 4 \\
\hline Algunas veces & 0 & 0 & 9 & 36 \\
\hline Rara vez o nunca & 0 & 0 & 5 & 20 \\
\hline Total & 25 & 100 & 25 & 100 \\
\hline \multicolumn{5}{|c|}{ Consumo de comida chatarra. } \\
\hline Todos los días & 1 & 4 & 0 & 0 \\
\hline $3-4$ veces por semana & 6 & 24 & 0 & 0 \\
\hline $1-2$ veces por semana & 6 & 24 & 0 & 0 \\
\hline Algunas veces & 10 & 40 & 5 & 20 \\
\hline Rara vez o nunca & 2 & 8 & 20 & 80 \\
\hline Total & 25 & 100 & 25 & 100 \\
\hline
\end{tabular}


Continuación Tabla 4.

\begin{tabular}{|c|c|c|c|c|}
\hline \multirow[t]{2}{*}{ Variables } & \multicolumn{2}{|c|}{ Antes de la Cx Bariátrica } & \multicolumn{2}{|c|}{ Después de la Cx Bariátrica } \\
\hline & Frecuencias & Porcentajes & Frecuencias & Porcentajes \\
\hline \multicolumn{5}{|c|}{ Consumo de carnes y pescado } \\
\hline Todos los días & 24 & 96 & 23 & 92 \\
\hline $1-2$ veces por semana & 0 & 0 & 1 & 4 \\
\hline Algunas veces & 1 & 4 & 0 & 0 \\
\hline Rara vez o nunca & 0 & 0 & 1 & 4 \\
\hline Total & 25 & 100 & 25 & 100 \\
\hline \multicolumn{5}{|l|}{ Consumo de harinas } \\
\hline Todos los días & 5 & 20 & 0 & 0 \\
\hline $3-4$ veces por semana & 8 & 32 & 1 & 4 \\
\hline 1-2 veces por semana & 1 & 4 & 0 & 0 \\
\hline Algunas veces & 9 & 36 & 2 & 8 \\
\hline Rara vez o nunca & 2 & 8 & 22 & 88 \\
\hline Total & 25 & 100 & 25 & 100 \\
\hline \multicolumn{5}{|l|}{ Consumo de dulces } \\
\hline Todos los días & 1 & 4 & 0 & 0 \\
\hline $3-4$ veces por semana & 11 & 44 & 0 & 0 \\
\hline 1-2 veces por semana & 4 & 16 & 1 & 4 \\
\hline Algunas veces & 8 & 32 & 5 & 20 \\
\hline Rara vez o nunca & 1 & 4 & 19 & 76 \\
\hline Total & 25 & 100 & 25 & 100 \\
\hline \multicolumn{5}{|c|}{ Consumo de frutas y verduras } \\
\hline Todos los días & 6 & 24 & 11 & 44 \\
\hline $3-4$ veces por semana & 6 & 24 & 9 & 36 \\
\hline $1-2$ veces por semana & 5 & 20 & 2 & 8 \\
\hline Algunas veces & 7 & 28 & 2 & 8 \\
\hline Rara vez o nunca & 1 & 4 & 1 & 4 \\
\hline Total & 25 & 100 & 25 & 100 \\
\hline
\end{tabular}

Tabla 5 Relación entre nivel de satisfacción con su condición física un año antes de la cirugía vs después de la cirugía en los pacientes obesos intervenidos con cirugía bariátrica en el Hospital Universitario Hernando Moncaleano Perdomo de la ciudad de Neiva.

Un año antes de la cirugía en qué medida se sentía satisfecho con su condición física

\section{Actualmente en qué medida se siente satisfecho con su condición física}

\begin{tabular}{|c|c|c|c|c|c|}
\hline Nada & $40 \%$ & $16 \%$ & $24 \%$ & $80 \%$ & \\
\hline Ligeramente & 0 & $16 \%$ & 0 & $16 \%$ & \\
\hline Moderadamente & 0 & 0 & $4 \%$ & $4 \%$ & 0,001 \\
\hline Total & $40 \%$ & $32 \%$ & $28 \%$ & $100 \%$ & \\
\hline
\end{tabular}

* Prueba Mc Nemar. 
Tabla 6 Relación entre medida en que se siente satisfecho con su vida un año antes de la cirugía Vs después de la cirugía en los obesos intervenidos a cirugía bariátrica en el Hospital Universitario Hernando Moncaleano Perdomo de la ciudad de Neiva.

\begin{tabular}{|c|c|c|c|c|c|c|}
\hline \multirow[b]{2}{*}{$\begin{array}{l}\text { Un año antes de la cirugía en qué } \\
\text { medida se sentía satisfecho con su vida }\end{array}$} & \multicolumn{4}{|c|}{$\begin{array}{l}\text { Actualmente en qué medida se siente } \\
\text { satisfecho con su vida }\end{array}$} & \multirow[b]{2}{*}{ Total } & \multirow[b]{2}{*}{$P^{*}$} \\
\hline & Ligeramente & Moderadamente & Bastante & Muy & & \\
\hline $\mathrm{Nada}$ & $4 \%$ & $24 \%$ & $20 \%$ & $20 \%$ & $68 \%$ & \\
\hline Ligeramente & 0 & 0 & $8 \%$ & 0 & $8 \%$ & \\
\hline Moderadamente & 0 & $4 \%$ & $12 \%$ & $8 \%$ & $24 \%$ & 0,001 \\
\hline Total & $4 \%$ & $28 \%$ & $40 \%$ & $28 \%$ & $100 \%$ & \\
\hline
\end{tabular}

* Prueba Mc Nemar.

Tabla 7 Distribución Funcionalidad de la Familia - APGAR. Los pacientes obesos intervenidos con cirugía bariátrica en el Hospital Universitario Hernando Moncaleano Perdomo de la ciudad de Neiva.

\begin{tabular}{lcc}
\hline & Frecuencia & Porcentaje \\
\hline Normo-funcional & 11 & 44 \\
Disfuncional- Leve & 14 & 56 \\
\hline Total & 25 & 100 \\
\hline
\end{tabular}

cirugía. Considero que este argumento también puede tener relación con los pacientes del estudio.

La mayoría de las personas que participaron en el estudio son de estrato socioeconómico 2 dos y esto posiblemente es por la naturaleza de la institución por ser el único hospital universitario de la región surcolombiana, el tipo de familia (nuclear, monoparental, extensa) de las personas que participaron en el estudio son familias nucleares y con un alto nivel de disfuncionalidad familiar leve teniendo esto relación con un estudio previos que señalan un predominio de familias nucleares (82\%) y disfuncionales el (54\%) ${ }^{[5]}$.

En el análisis de ingesta alimentaría en nuestro estudio podemos observar que los participantes presentan una alta ingesta de atracones de comida, ingesta hipercalórico y de alimentos grasos, también podemos observar que antes de la cirugía se presenta un alto consumo de alimentos como harinas, dulces, comida chatarra, grasas de origen animal. Este comportamiento puede estar relacionado con lo que propone Kaplan y Kaplan ${ }^{[6]}$ consideran la sobre ingesta como una conducta aprendida, utilizada por el sujeto obeso como mecanismo para reducir la ansiedad. Según Ambrosi ${ }^{[7]}$ considera que actualmente la sociedad parece converger en malos hábitos alimenticios, optado por dietas pocos saludable (comidas rápidas) y abandonando la actividad física, estas son algunas de las principales causas de obesidad.

Las diferentes comorbilidades que más se presentaron en las personas que participaron en el estudio fue la gastritis, seguido de la diabetes, la HTA, artrosis, según la OMS El sobrepeso y la obesi- dad son el quinto factor principal de riesgo de defunción en el mundo. Cada año fallecen por lo menos 2,6 millones de personas adultas como consecuencia del sobrepeso o la obesidad, es la generadora de enfermedades como hipertensión arterial, enfermedades articulares, diabetes, alteraciones respiratorias, deterioro de la función renal, hepática entre otras como también la depresión, trastornos de la conducta alimentaria y alteración de la calidad de vida.

Se considera que la familia desempeña un papel importante en la salud de cada uno de sus integrantes; cuando la familia pierde su capacidad de funcionar, ocurren alteraciones en el proceso de salud enfermedad de todos sus miembros ${ }^{[8]}$. Según lo expresado por los participantes del estudio el $56 \%$ en la evaluación del APGAR Familiar presentan disfunción familiar leve ósea que prestan dificultades en núcleo familiar por todo lo anterior puede coincidir con lo expuesto por López y Macilla ${ }^{[5]}$ que argumentan que la familia juega un papel determinante en la génesis de los problemas alimenticios y la obesidad puede ser proporcionada por una dinámica familiar disfuncional, en donde el individuo obeso funciona como un medio para aminorar los conflictos y tensiones que se propician por dicha dinámica el sobrepeso está directamente correlacionado con una alta problemática familiar y la obesidad de los niños y niñas se encuentra asociada con la salud mental de otros miembros de la familia y con el funcionamiento de ésta por lo tanto, las familias obesas poseen una dinámica que se asemeja a la de las familias psicosomáticas.

Dentro de los resultados observados un factor importante es el nivel de satisfacción que tenía la persona de su condición física antes de la cirugía y actualmente según lo manifestado por los pacientes el más alto porcentaje consideraba que para nada se sentían satisfecho con su condición física después de la cirugía bariátrica consideran que moderadamente se sienten satisfechos con su condición física, cabe aclarar que la condición física puede presentar alteraciones significativas para el obeso debido a que se le dificulta realizar actividades como caminar, amarrarse los zapatos, bañarse, vestirse, danzar entre otras actividades. Considero que nuestros resultados puede tener relación con lo expresado por Nguyen ${ }^{[9]}$ argumenta que la función física de los pacientes están significativamente alterada antes de someterse a un bypass gástrico abierto o laparoscopio. En las evaluaciones a los 3 y 6 meses después de la intervención, la función física había mejorado. 
En cuanto al nivel de satisfacción de su condición de vida un año antes de la cirugía una alta proporción argumenta que no se sentían para nada satisfechos en la actualidad un alto porcentaje consideran sentirse moderadamente satisfechos, Considero que los datos obtenidos pueden estar haciendo referencia con lo argumentado por Wadden ${ }^{[10]}$ que considera que la obesidad extrema casi siempre disminuye la calidad de vida relacionada con la salud.

Considero que el nivel de satisfacción con la vida y el nivel de satisfacción en condición física en este momento de la vida ósea después la cirugía se esperaría que hubiera sido mucho más alto el nivel de satisfacción y este argumento puede tener relación con lo expresado según Powers ${ }^{[11]}$ argumentan que "mientras la cirugía bariátrica es un método efectivo para la pérdida de peso para muchos pacientes, no es la cura para los problemas de autoestima”. En mi opinión personal, la cirugía bariátrica es una de las muchas herramientas disponibles para ayudar a las personas a llegar a su estado de salud físico que se desea. Y de esta manera puede liberarlo para prestar más atención a los problemas relacionados a su salud mental. La cirugía bariátrica no podrá llenar los vacíos del corazón, ni del alma pero muy posible podrá acercarnos a un lugar en que nos sintamos listos para trabajar en ese tipo de sanidad

\section{Conclusiones}

Las personas que se realizaron la cirugía bariátrica en el Hospital Universitario Hernando Moncaleano Perdomo de Neiva de septiembre de 2011 a septiembre de 2012, se caracterizan por ser de sexo femenino, en edades entre 20 y 53 años que cuentan con pareja estable de estrato socioeconómico bajo que se desempeñan en el hogar con un nivel de escolaridad hasta secundaria y viven en Neiva.

El peso de las personas antes de la cirugía estaba entre $93 \mathrm{Kg}$ y $155 \mathrm{Kg}$. con un IMC entre 36 y 59, con un alto porcentaje de diferentes comorbilidades además los controles postoperatorio de asistencia que más registros presentan es al tercer mes.

Las personas que se intervinieron presentan antecedente familiares de sobrepeso (padres, hermanos e hijos) como también diferentes comorbilidades como la misma obesidad, diabetes, HTA entre otros. Antes de la cirugía los pacientes presentan malos hábitos alimenticios llevándolos al punto de presentar una ingesta alimentaría del gran comilón que está relacionado con la alta ingesta de atracones de comida, después de la cirugía se puede evidenciar el decrecimiento de la alta ingesta de alimentos llevándolos a buenos hábitos alimenticios. Cabe resaltar que la mayoría de las personas no presentan la conducta de fumar o de consumir licor en su vida.

Es importante mencionar que las personas que se realizaron la cirugía tienen una familia nuclear (papás e hijos) además presentan disfuncionalidad familiar leve. E1 80\% que consideraba que antes de la cirugía para nada se sentía bien con su condición física en la actualidad el $40 \%$ se sienten moderadamente satisfechos y el nivel de satisfacción con su vida antes de la cirugía el $68 \%$ consideraba sentirse para nada satisfechos con su vida actualmente el $24 \%$ considera que se sienten moderadamente satisfechos, la cirugía bariátrica ha evidenciado un cambio físico que genera impacto en los participantes del estudio en cuanto al nivel de satisfacción con la condición física y de su vida se evidencia un cambio pero se esperarían un impacto de mayor proporción.

Cabe aclarar que las características de la muestra (intencional, no probabilística) las conclusiones que se desprenden de este trabajo son válidas únicamente para describir al grupo de sujetos que participaron en esta investigación.

\section{Bibliografía}

1. Salud, O.M.D.I., Obesidad y sobrepeso. Centro de prensa, Nota descriptiva N $N^{\circ} 31$ 1. Consultado: marzo de 2012.

2. Escobar, I. and A.B. Rossi, Sobrepeso y obesidad incrementan la epidemia de diabetes tipo 2. Consultado: junio de 2012.

3. Harraca, J.L., Obesidad · Aspectos Generales. Consultado: marzo de 2012.

4. Lic. Jessica A. Erickson, D.J.R., La Relación entre Obesidad y Salud Mental. La Relación entre Obesidad y Salud Mental. Departamento de Nutrición, Diabetes y Metabolismo. Facultad de Medicina. Pontificia Universidad Católica de Chile.

5. Rojas López, J.P., Influencia de la disfunción familiar y factores sociales, en la prevalencia de obesidad/desnutrición. 2010.

6. Gil, G.P. and R. Gálvez, Medicina preventiva y salud pública. 1992: Ediciones Científicas y Técnicas.

7. Ambrosi, E.C., Bienestar psicológico y sentido de vida en adultos obesos (Tesina de grado). Mendoza, Universidad del Aconcagua. Facultad de Psicología., 2009.

8. Dorado-Gaspar, M., J. Loría-Castellanos, and M. Guerrero-Martínez, Aptitud clínica hacia el estudio integral de la familia en residentes de medicina familiar. Archivos en Artículo Original Vol, 2006. 8(3):169-174.

9. Nguyen, N.T., et al., Laparoscopic versus open gastric bypass: a randomized study of outcomes, quality of life, and costs. Ann Surg, 2001. 234(3):279-89.

10. Wadden, T.A., et al., Treatment of obesity by behavior therapy and very low calorie diet: a pilot investigation. J Consult Clin Psychol, 1984. 52(4):692-4.

11. Mitchell, J.E. and M. De Zwaan, Bariatric Surgery: A Guide for Mental Health Professionals. 2005: Routledge. 